\title{
Protective Immunization of monkeys with BCG or BCG plus heat-killed Mycobacterium leprae: clinical results
}

\author{
BOBBY J. GORMUS, GARY B. BASKIN, KEYU XU, \\ RUDOLF P. BOHM, PAMELA A. MACK, \\ MARION S. RATTERREE, SANG-NAE CHO*, \\ WAYNE M. MEYERS $\dagger \&$ GERALD P. WALSH $\ddagger$ \\ Departments of Microbiology, Pathology and Veterinary Sciences, \\ Tulane Regional Primate Research Center, 18703 Three Rivers Road, \\ Covington, LA 70433, USA; *Department of Microbiology, Yon-Sei \\ University School of Medicine, Seoul, Republic of Korea; †Armed \\ Forces Institute of Pathology, Washington, DC; and $¥$ American \\ Leprosy Foundation, Rockville, MD, USA
}

\section{Accepted for publication 7 November 1997}

\begin{abstract}
Summary Rhesus and sooty mangabey monkeys (RM and SMM) were vaccinated and boosted with BCG or BCG + low dose (LD) or high dose (HD) heat-killed Mycobacterium leprae (HKML). One group was not vaccinated. Except for a group of controls, all monkeys were challenged with live M.leprae. All animals were studied longitudinally to determine antileprosy protective efficacy. BCG reduced the numbers of RM with histopathologically-diagnosed leprosy by $70 \%$ and slowed and ameliorated the appearance of symptoms. BCG + LDHKML reduced the number of RM with leprosy by $89 \%$ and BCG + HDHKML by $78 \%$. BCG did not protect SMM from developing leprosy, but disease progress was slowed; disease in SMM was exacerbated by the addition of HKML to the vaccine. RM, as a species, are prone to paucibacillary (PB) forms of leprosy, whereas SMM are prone to multibacillary (MB) forms. Thus, BCG vaccination offers significant protection from clinical disease and slows/ameliorates the rate of progression/degree of disease at the PB end and appears to at least ameliorate symptoms at the $\mathrm{MB}$ end of the leprosy spectrum. $\mathrm{BCG}+\mathrm{HKML}$ protects at the PB end and exacerbates disease progress at the MB end of the leprosy spectrum.
\end{abstract}

\section{Introduction}

There have been numerous field trials in various human populations to determine the antileprosy immunoprophylactic efficacy of Mycobacterium bovis, strain bacillus Calmette-Guerin (BCG) alone or in combination with killed M. leprae. Five controlled ${ }^{1-5}$ and two case-control or cohort studies ${ }^{6,7}$ have been reported using BCG alone, with striking 
variations in results. Protective rates using BCG alone ranged from near 20 to $80 \%$. The Venezuelan study included subjects immunized with heat-killed M. leprae (HKML) plus $\mathrm{BCG}^{2}$ This latter study concluded that there was no difference between BCG alone $v s$ BCG in combination with HKML in protective efficacy. The study was difficult to clearly interpret, however, because no placebo group was included and the results were of marginal statistical significance. ${ }^{2}$ Thus, many questions remain unanswered concerning the ability of either BCG alone or $\mathrm{BCG}+$ killed $M$. leprae to impart protection against clinical leprosy. Reasons for the wide differences in the effectiveness of BCG alone in leprosy protection from one study to another also remain to be understood.

Several explanations have been proposed as possible reasons for the variations in results observed in the efficacy of BCG to protect against leprosy. ${ }^{6}$ One suggestion is that prior exposure to differing types of mycobacteria in the environment may differentially alter the susceptibility of individuals in different parts of the world to infections with M.leprae and/or alter the responses to mycobacteria-containing vaccines. ${ }^{8,9}$ Another possibility is that BCG preparations from different sources and/or batches or lots were used in many of the reported studies, which might produce variations in immunizing potency. ${ }^{10,11}$ Differences in natural history of infection and disease, such as variations in the ratios of tuberculoid (TT) to lepromatous (LL) forms observed in populations in different parts of the world or differences in population genetics have also been suggested as explanations. ${ }^{6}$

In the present studies, many, if not all, of these weaknesses were controlled or eliminated. All of the monkeys were born, reared and maintained in the southern United States, were similar to one another in age and were randomized among our groups according to sex. The same BCG (Glaxo) lots and concentrations were used in all cases; the M. leprae used for immunization was all from a single purified and heat-killed batch; and the live M. leprae used to challenge the animals was all from a single source. Another important aspect of these studies is that rhesus monkeys (RM), as a species, tend ( $>80 \%)$ to develop TT forms, (Gormus, BJ et al, p. 24) whereas sooty mangabey monkeys (SMM) tend (>80\%) to develop LL forms of leprosy. ${ }^{12-15}$ Thus, the relationship between natural history of disease and immunizability can be examined directly by comparative vaccine studies between RM $v s$ SMM.

\section{Methods}

ANIMALS

Forty-five Chinese rhesus monkeys (RM) (Macaca mulatta), 2-3 years old, born and maintained in our breeding colony at the Tulane Regional Primate Research Centre (TRPRC), all with presumed similar natural exposures to environmental agents, were divided into 4 experimental groups (3 vaccine groups and 1 unvaccinated control group-all M. leprae challenged) of 10 ( 3 females and 7 males/group). There was also one group of 5 unvaccinated, non- $M$. leprae challenged normal controls (Table 1).

Thirty-five sooty mangabey monkeys (SMM) (Cercocebus torquatus atys), aged 3-10 years, were purchased from the Yerkes Regional Primate Research Center's breeding colony, Atlanta, GA, USA (where they were born and reared). The SMM were divided into 4 $M$. leprae-challenged experimental groups and one normal, unchallenged control group of 7 animals per group (2 females and 5 males/group) (Table 1). 
Table 1. Monkey immunizations

\begin{tabular}{lcccccc}
\hline Group & \#RM & \#SMM & $\begin{array}{c}\text { Total \# } \\
\text { Monkeys }\end{array}$ & BCG* & $\begin{array}{c}\text { HKML } \\
\text { Dose* }\end{array}$ & $\begin{array}{c}\text { M. leprae } \\
\text { Inoc }\end{array}$ \\
\hline 1 & 10 & 7 & 17 & No & 0 & Yes \\
2 & 10 & 7 & 17 & Yes & 0 & Yes \\
3 & 10 & 7 & 17 & Yes & $1 \cdot 6 \times 10^{9}$ & Yes \\
4 & 10 & 7 & 17 & Yes & $16 \times 10^{9}$ & Yes \\
5 & 5 & 5 & 10 & No & 0 & No \\
\hline
\end{tabular}

Abbreviations: RM, rhesus monkeys; SMM, sooty mangabey monkeys; BCG, Bacillus Calmette-Guerin, Glaxo (present manufacturer, Evans Medical, UK); Inoc, inoculation with live M. leprae.

*Monkeys were boosted with the same doses of BCG or BCG + HKML as those used for vaccination; RM were boosted 5 weeks and SMM 7 weeks post-vaccination.

\section{SELECTION OF BCG}

Freeze-dried BCG was purchased from three sources: 1, Glaxo strain (ST1077) from Evans Medical Limited, Liverpool, England; 2, strain \#1331 (batch 590) from the Staatens Seruminstitute, Copenhagen, Denmark; and 3, Merieux strain from Connaught, Inc., Willowdale, Ontario, Canada.

We obtained a single lot of each of the Copenhagen and the Merieux BCG's, whereas we had on hand a quantity of ampoules of BCG from the Glaxo Company, Middlesex, England and obtained a new shipment of the Glaxo strain from Evans Medical, Liverpool, which presently owns the Glaxo Company. The Evans shipment included multiple lot \#'s. Thus, we used mixtures of several lot \#'s of the Glaxo strain for preliminary testing and the same mixtures for all immunizations and boosting. BCG's were stored at $2^{\circ}-8^{\circ} \mathrm{C}$ wrapped in foil to protect from light. BCG's were checked for viability prior to use (see below). The following Glaxo lot \#'s were utilized: G4386DA, D428, W1533A (Evans), E2528A (Evans).

BCG from each of the 3 sources was tested by intracutaneous (IC) injection of $0 \cdot 1 \mathrm{ml}$ $\left(10^{6}-10^{7}\right.$ viable units) into $2 \mathrm{RM}$. The resulting reactions at $2-3$ weeks postvaccination (PV) were in excess of $1-\mathrm{cm}$ diameter induration with ulceration at both Glaxo sites, $5 \mathrm{~mm}$ and negative at the two Copenhagen sites, respectively, and negative at both Merieux sites. Fortyeight-hour TB test results of the six RM at 3 weeks PV gave positive results in the same $3 \mathrm{RM}$ ( 2 Glaxo and 1 Copenhagen BCG recipient) and negative results in the other three RM. Boosting with the respective three BCG's at 4 weeks PV gave approximately $1-\mathrm{cm}$ erythematous indurations in the four Glaxo and Copenhagen RM's and 1- and $1 \cdot 5-\mathrm{cm}$ ulcerated indurations in the two Merieux recipients at 48-hr postboosting. Based on these observations, we chose the Glaxo BCG for use in the present vaccine studies.

\section{PREPARATION OF HEAT-KILLED M.LEPRAE (HKML) FOR VACCINATION}

In 1988, two armadillos were inoculated at the Armed Forces Institute of Pathology (AFIP) with SMM-origin M. leprae isolated from lepromatous nodules from a SMM (G930); a third armadillo was inoculated with M. leprae from a second SMM (D172). Both G930 and D172 had been experimentally inoculated with $M$. leprae taken originally from a SMM (A015) with naturally-acquired leprosy. ${ }^{12,16}$ Livers and spleens were taken from the three armadillos when 
leprosy became sufficiently advanced and were stored frozen $\left(-70^{\circ} \mathrm{C}\right)$ until shipment to the laboratory of Dr Patrick J. Brennan (Department of Microbiology, School of Veterinary Medicine, Colorado State University, Fort Collins, CO, USA) for isolation and purification of M. leprae by the Draper method. ${ }^{17} \mathrm{~A}$ total of $735 \mathrm{~g}$ of liver and $257 \mathrm{~g}$ of spleen all at approximately $10^{9} \mathrm{M}$. leprae/g of tissue was shipped to Dr Brennan. A portion of one liver $(47.89 \mathrm{~g})$ yielded $228 \mathrm{mg}$ of G930 M. leprae (approximately $6.6 \times 10^{11}$ organisms) and $47.85 \mathrm{~g}$ of a second liver yielded $190 \mathrm{mg}$ of D172 M. leprae (approximately $5.5 \times 10^{11}$ organisms). The M.leprae preparations were heat-killed (autoclaved), lyophilized and shipped to the TRPRC for monkey immunizations. These procedures were performed by Dr Brennan's laboratory under contract \#AI-52582 from the National Institute of Allergy and Infectious Diseases.

IMMUNIZATIONS WITH BCG OR BCG PLUS HKML

Monkeys were vaccinated with BCG alone or BCG + high-dose (HD) HKML or BCG + low-dose (LD) HKML by IC injection of $0.1 \mathrm{ml}$ of the appropriate suspension. HKML was weighed so that LD tubes contained $0 \cdot 275 \mathrm{mg}$ of $M$.leprae from each of two armadillos, i.e. one inoculated with $M$. leprae from SMM D172 and another inoculated from SMM G930, for a total HKML dose of $0.55 \mathrm{mg}\left(1.6 \times 10^{9} \mathrm{HKML}\right)$. The HD tubes contained twice as much of each of the two M.leprae preparations for a total of $1.1 \mathrm{mg}$ or $3.2 \times 10^{9}$ HKML. Immediately prior to use, Glaxo BCG was reconstituted to $10-26 \times 10^{6}$ viable units/ $\mathrm{ml}$ with sterile distilled water (or, in the case of the Evans Medical-origin Glaxo strain, with sterile saline, as recommended by the manufacturer) and was placed in ice in the dark after reconstitution; $0 \cdot 1 \mathrm{ml}$ was used as such for BCG-only recipients or was mixed with a LD or HD HKML tube using a $0 \cdot 5-\mathrm{ml}$ insulin syringe, the suspension was reaspirated into the syringe and the suspension injected into a single site in the dorsal scapula area of each monkey. All 3 vaccine groups received the same dose of BCG. Table 1 shows the monkey groupings for immunizations and boosting and the amounts of BCG or BCG + HKML used for immunization and for boosting. Primary vaccinations were carried out on day 0; boosting at $5(\mathrm{RM})$ or 7 (SMM) weeks; and challenge with live M. leprae was at $10(\mathrm{RM})$ or 15 (SMM) weeks.

BCG viability and growth was confirmed by Dr Thomas M. Schinnick, Division of Bacterial and Mycotic Diseases, Centers for Disease Control, Atlanta, GA, USA. At least $30 \%$ viability was confirmed in ampoules from all lot numbers, but exact viable counts could not be determined due to clumping.

\section{PREPARATION OF LIVE ML FOR CHALLENGE}

In September, 1991, armadillos were inoculated at the AFIP with M.leprae taken from lepromatous (LL) dermal nodules of SMM D173, which had been subinoculated from a case of natural leprosy in SMM A015 $5^{18}$ and had been subpassaged through SMM A022 prior to inoculation into D173. ${ }^{13}$ For M. leprae inoculations (monkey challenge), one of these armadillos was sacrificed (on 26/5/91 for RM challenge and another on 8/9/92 for SMM challenge); LL nodules, spleen and liver were harvested and shipped overnight on ice to the TRPRC where each tissue was processed separately for M.leprae isolation by methods previously published. ${ }^{13}$ Briefly, tissues were cut into small pieces, fat removed and pieces minced and homogenized in cold phosphate-buffered saline using a Dounce homogenizer 
with a 40 MI mortar and Teflon pestle (Wheaton Scientific, Millville, NJ, USA), passed through gauze and centrifuged at $200 \times \mathbf{G}$ for $5 \mathrm{~min}$. at $4^{\circ} \mathrm{C}$. The acid-fast bacilli (AFB) in the supernatant were counted and morphologic indices (MI) determined by the method of Shepard \& McCrae. ${ }^{19}$

\section{MONKEY INOCULATIONS}

Monkeys were inoculated with M. leprae suspensions by combined IC and intravenous (IV) routes using 2 IC sites per ear, the tip of the nose, outer forearms and outer calves. IV inoculations were made via the saphenous vein. Details have been previously published. ${ }^{13}$ M. leprae suspensions for RM challenge had an MI of $8 \%$ and for SMM MI $=10 \%$.

\section{CLINICAL OBSERVATIONS}

Animals were observed daily and examined in detail 3-4 times per year or more, depending on the status of the animal, and the clinical aspects of the disease were recorded. The RidleyJopling system was used to classify leprosy immunohistopathologically, ${ }^{20}$ with the exception that classification at the paucibacillary (PB) end of the spectrum differs slightly in RM from humans. The classification Ind/TT (Indeterminate/tuberculoid) describes a lesion with rare to few AFB, containing epithelioid and/or multinucleated giant cells, but with fewer lymphocytes than usually seen in human TT leprosy (Gormus, BJ, et al. p. 24). Nasal smear samples were taken on microscope slides at each time of observation for staining with Ziehl-Neelsen for the presence of AFB in nasal secretions.

\section{HISTOPATHOLOGIC DETERMINATIONS}

Biopsies were taken at intervals from dermal inoculation sites and/or from sites of dissemination for analysis of the lesions and for documentation of clinical leprosy after staining with H\&E and Fite-Faraco, as previously reported. ${ }^{21,22}$ A diagnosis of leprosy was made on the basis the presence of acid-fast bacilli (AFB): 1, in nasal secretions (since we have previously determined that the presence of AFB in such secretions is the first indication of dissemination of leprosy beyond areas of dermal inoculation; and/or 2, within areas of inflammatory infiltration at inoculation sites at a time when clearance of AFB had occurred in a significant number of animals; or 3, in disseminated dermal sites (that had not been inoculated with M.leprae). Leprosy was classified histopathologically according to the Ridley-Jopling system, ${ }^{20}$ with the exception that at the PB end of the spectrum differ slightly in RM from humans, as previously noted (Gormus BJ et al, p. 24). For purposes of this report, however, results are given as PB or MB. MB will include borderline to lepromatous forms on the Ridley-Jopling spectrum; ${ }^{20} \mathrm{~PB}$ includes indeterminate, tuberculoid and borderline tuberculoid forms.

\section{DETECTION OF SERUM PGL-I ANTIGEN LEVELS}

Serum PGL-I levels were detected in serum samples by a dot-ELISA method; results were expressed as $0 ;+/-(0 \cdot 5)$ and $1+$ to $4+$, as previously described. ${ }^{12,23}$ 
STATISTICAL ANALYSES

All statistical calculations were performed using statistical programs for the Macintosh Computer. Longitudinal comparisons between groups were performed by Mancova analysis except for the cumulative numbers of animals with disease $v s$ time data. The latter statistical comparisons were performed by Fisher's exact method at each time point.

\section{Results}

REACTIONS TO IMMUNIZATIONS WITH BCG OR BCG + HKML

In $\mathrm{RM}$, reactions at the sites of vaccination peaked within 1-2 weeks and averaged about $17 \mathrm{~mm}$ in diameter for BCG + HKML and $8 \mathrm{~mm}$ for BCG alone (Figure 1(a)); reactions to

A.

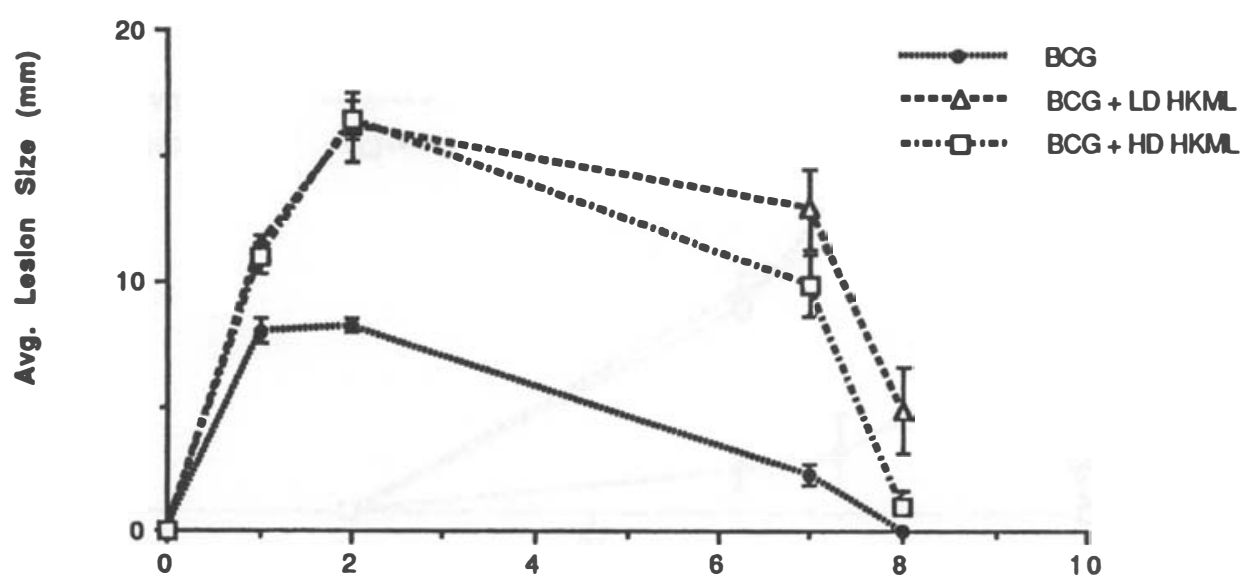

B.

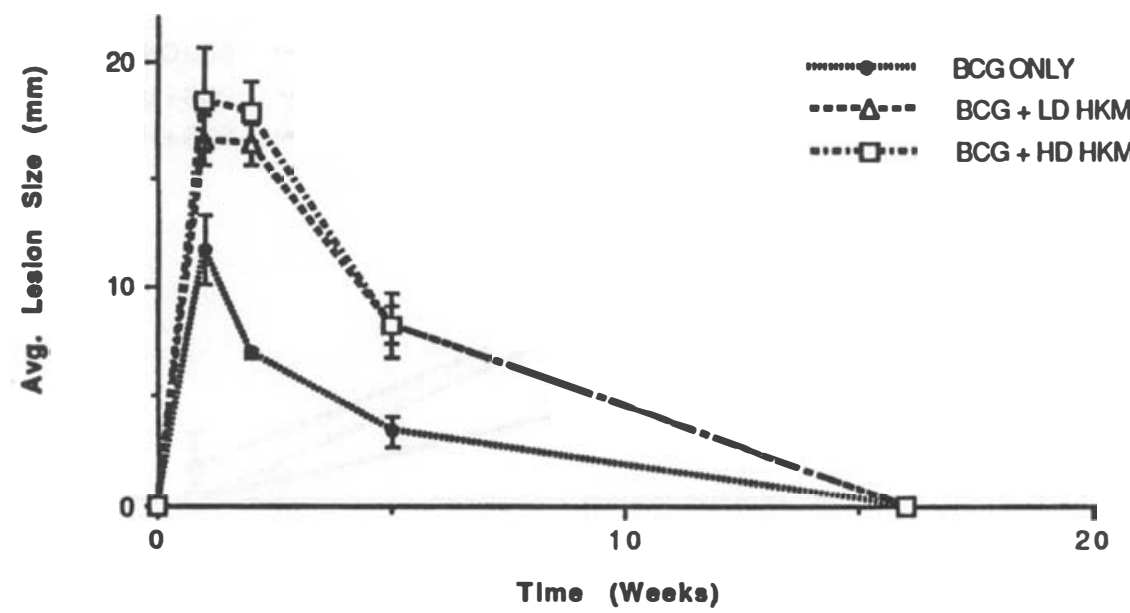

Figure 1. Average reaction sizes over time $(+/-1$ standard error of the mean, SEM) at dermal sites of primary immunization (a) or boosting (b) in RM (10/group). 
boosting peaked within 1 week near $17.5 \mathrm{~mm}$ for BCG + HKML and $12 \mathrm{~mm}$ for BCG alone (Figure 1(b)). In SMM, reactions to vaccination also peaked within 1-2 weeks and averaged between 10 and $15 \mathrm{~mm}$ for BCG + HKML and 5.5 mm for BCG alone (Figure 2(a)); reaction sizes similar to those resulting from vaccination were observed within 2 weeks postboosting (Figure 2(b)).

\section{CLINICAL OBSERVATIONS IN RM AFTER CHALLENGE WITH LIVE M. LEPRAE}

The clinical results for RM are shown in Table 2 and graphically in Figure 3 and are described in detail below. RM as a species are more resistant than SMM to leprosy; on the average, more than $80 \%$ of RM develop PB forms of experimental leprosy, depending on the

A.

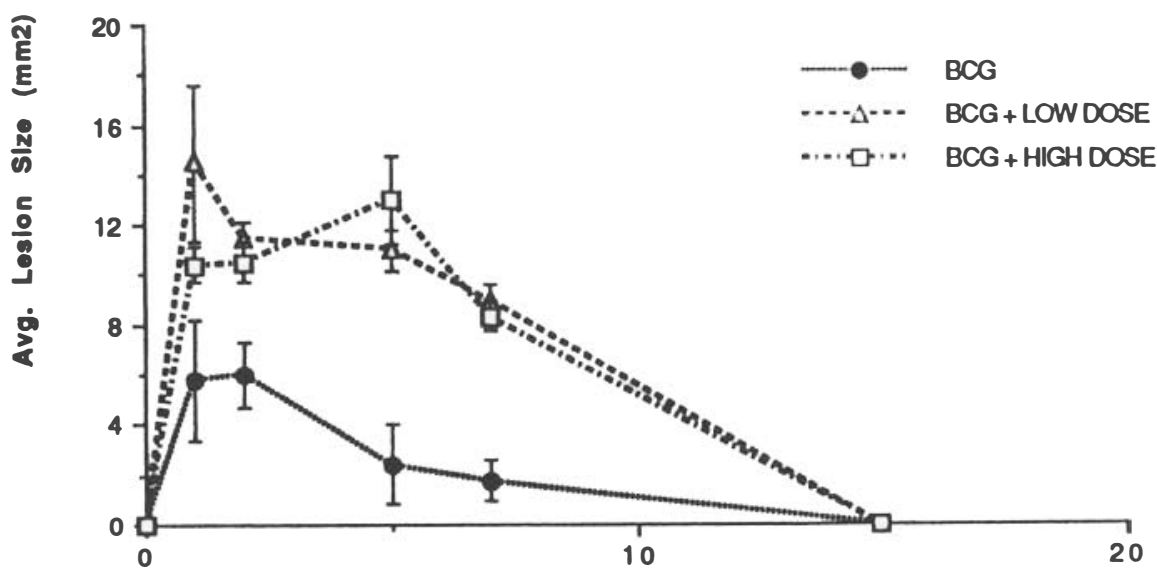

B.

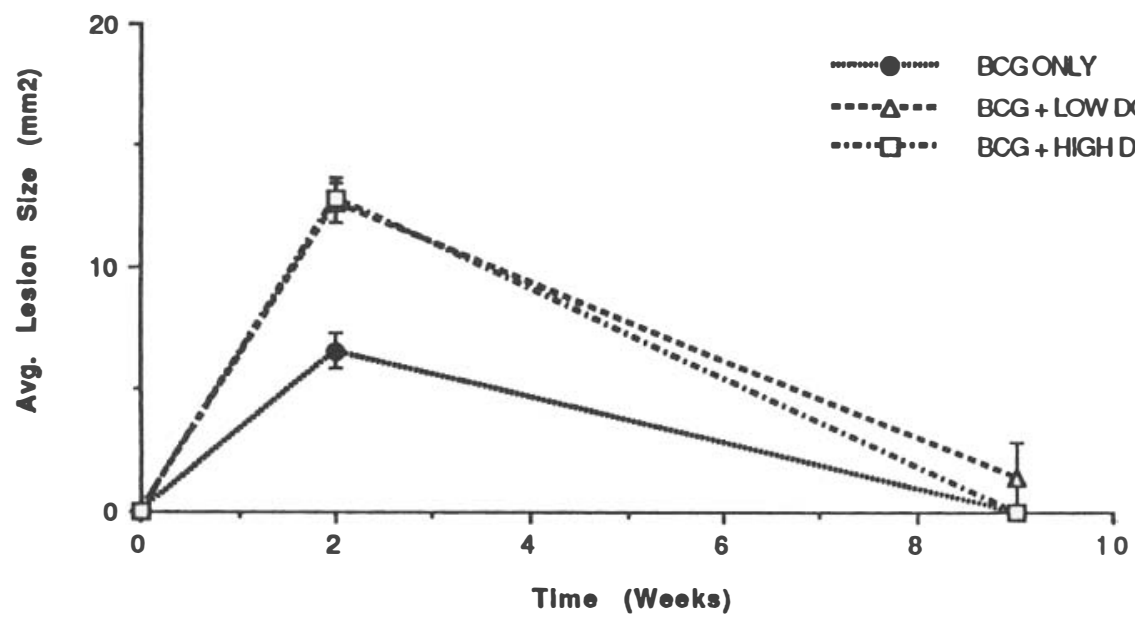

Figure 2. Average reaction sizes over time (+/- 1 SEM) at dermal sites of primary immunization (a) or boosting (b) in SMM (7/group). 
experiment (Gormus, BJ et al, p. 24). In the experiments to be described herein, larger than usual numbers of unvaccinated, control RM developed leprosy, presumably due to high MI's, the quality of the inoculum and the numbers of ML inoculated (Table 2; Figure 3). By 1198 days PI, 9 of 10 unvaccinated RM had developed biopsy-positive leprosy compared to 3 BCG only, 1 in BCG + LDHKML and 2 in the BCG + HDHKML vaccinated groups (Figure 3). These numbers did not increase over the subsequent period of observation (more than 1500 days).

In RM, within the first 2-3 months postinoculation (PI), reactions and clinical appearances of leprosy at dermal sites of inoculation might be misleading due to the local injection of relatively large numbers of M. leprae.$^{14,15}$ By 3 months PI, most leprosy-resistant RM have cleared the AFB from dermal inoculation sites (Table 2 and Gormus, BJ et al, p. 24). Thus, we have focused below on results beginning at 91 days PI and thereafter in RM.

\section{UNVACCINATED GROUP}

We will publish separately a detailed accounting of the histopathologic results of these studies (Baskin, GB et al, manuscript in preparation). For the present purposes, histopathologic results will be given only as PB vs MB. During the 91-330 day period PI, the unvaccinated group of RM showed the following (Table 2): one RM (K576) had AFB in nasal secretions, K656 had PB leprosy at dermal inoculation sites, L124 had PB at inoculation sites and K971 had disseminated (Dssm) MB leprosy at dermal sites. During that period, two of the remaining $6 \mathrm{RM}$ did not show histopathologically-documentable leprosy and 4 did not have lesions warranting biopsy. Nine of the 10 unvaccinated RM (positive controls) developed clinical signs of leprosy with solidly-staining AFB documentable in biopsies and nasal secretions during the period of observation reported upon herein (1542 days). It was necessary to treat seven of the 10 unvaccinated RM.

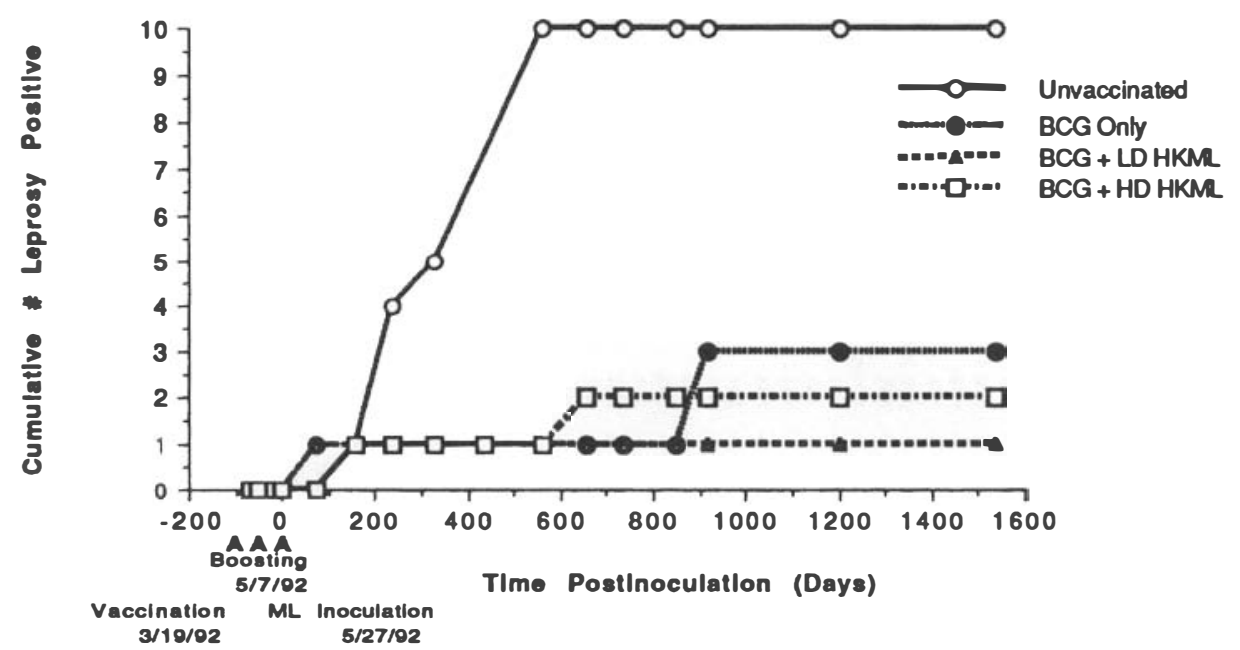

Figure 3. Cumulative number of leprosy-positive RM, based on biopsy results Ridley-Jopling classifications of infiltrations of AFB-positive dermal lesions, in the unvaccinated or vaccinated groups over the course of observation after live $M$. leprae challenge. 


\begin{tabular}{|c|c|c|c|c|c|c|c|c|c|c|c|c|c|c|c|c|c|c|c|c|c|c|c|c|c|c|}
\hline \multirow[t]{2}{*}{ Days PI } & \multicolumn{6}{|c|}{$91-330$} & \multicolumn{5}{|c|}{$331-560$} & \multicolumn{5}{|c|}{$561-850$} & \multicolumn{5}{|c|}{$851-1197$} & \multicolumn{5}{|c|}{ 1198-1542 } \\
\hline & & $\mathrm{N}-\mathrm{AFB}$ & Dssm & $\mathrm{Bx}$ & $\mathrm{Rx}$ & Notes & $\mathrm{N}-\mathrm{AFB}$ & Dssm & $\mathrm{Bx}$ & $\mathrm{Rx}$ & Notes & $\mathrm{N}-\mathrm{AFB}$ & Dssm & $B x$ & $\mathrm{Rx}$ & Notes & $\mathrm{N}-\mathrm{AFB}$ & Dssm & $\mathrm{Bx}$ & $\mathrm{Rx}$ & Notes & N-AFB & Dssm & $\mathrm{Bx}$ & $\mathrm{Rx}$ & Notes \\
\hline \multirow[t]{10}{*}{ Group I } & K131 & - & - & ND & - & reg & - & - & ND & - & reg & $1+$ & - & ND & - & prog & - & - & ND & - & reg & $4+$ & - & ND & - & slow prog. \\
\hline & K262 & - & - & - & - & NVL & - & - & PB & - & prog & $1+$ & - & ND & - & prog & - & + & PB & - & prog & $4+$ & + & ND & + & prog \\
\hline & K574 & - & - & ND & - & NVL & - & - & ND & - & NVL & - & + & PB & - & prog & $4+$ & + & PB & - & prog & - & + & PB & + & nc, prog \\
\hline & K576 & $4+$ & - & ND & - & prog & $4+$ & + & MB & - & ENL, prog & $4+$ & + & MB & - & prog & $2+$ & + & MB & + & prog & - & + & ND & + & $\mathrm{nc}$, healing \\
\hline & K656 & - & - & PB & - & prog & - & - & ND & - & resis & - & - & - & - & resis & - & - & ND & - & resis & - & - & ND & - & resis \\
\hline & K941 & - & - & ND & - & prog & - & - & $\mathrm{ND}$ & - & static & $4+$ & - & ND & - & st, prog & - & - & PB & - & resis & - & - & ND & - & st, resis \\
\hline & K966 & - & - & - & - & NVL & - & - & ND & - & static & $3+$ & - & ND & - & st, prog & $4+$ & - & ND & - & st, prog & - & - & PB & + & resis \\
\hline & K971 & - & + & MB & - & prog. suscep & $4+$ & + & MB & - & prog & - & + & ND & - & static & $4+$ & + & MB & + & prog, suscep & - & + & MB & + & st, healing on $\mathrm{Rx}$ \\
\hline & K975 & - & - & $\mathrm{PB}$ & - & NVL & $1+$ & - & PB & - & static & $4+$ & - & ND & - & st, prog & - & + & neuritic PB & + & $\mathrm{st}, \mathrm{nc}$ & ND & ND & ND & + & $\mathrm{sac}$ \\
\hline & L124 & - & - & PB & - & st, prog & - & + & PB & - & prog & - & + & ND & - & st, static & - & + & ND & - & st, static & - & + & neuritic PB & + & nc, st, prog \\
\hline \multirow[t]{10}{*}{ Group II } & K452 & - & - & ND & - & NVL & $1+$ & - & ND & - & prog & $4+$ & - & ND & - & prog & $2+$ & - & ND & - & NVL & - & - & ND & - & reg \\
\hline & K477 & - & - & ND & - & NVL & - & - & $\mathrm{ND}$ & - & NVL & $4+$ & - & ND & - & prog & - & - & ND & - & st, reg & - & - & ND & - & st, reg. sac \\
\hline & K700 & - & - & ND & - & NVL & - & - & $\mathrm{ND}$ & - & NVL & - & - & ND & - & NVL, resis & - & - & ND & - & NVL & - & - & ND & - & NVL \\
\hline & K833 & - & - & ND & - & NVL & - & - & ND & - & NVL & - & - & ND & + & debil, wt loss & - & - & ND & + & On $\mathrm{Rx}$ & - & - & ND & + & healing on $R x$ \\
\hline & K948 & - & + & ND & - & prog & $4+$ & + & ND & - & prog & - & - & ND & - & static & - & - & PB & - & st, reg & - & - & ND & - & static \\
\hline & K968 & - & - & ND & - & NVL & - & - & ND & - & NVL & - & - & ND & - & NVL & - & - & ND & - & NVL & - & - & $\mathrm{ND}$ & - & NVL \\
\hline & L060 & - & - & MB & - & static & - & - & - & - & reg & $4+$ & - & - & - & prog & - & - & ND & - & reg & - & - & neuritic MB & + & $\mathrm{nc}, \mathrm{sac}$ \\
\hline & L123 & - & - & ND & - & NVL & - & - & ND & - & NVL & $3+$ & - & ND & - & prog & - & - & ND & - & st, reg & - & - & prob.neuritic PB & - & $\mathrm{nc}$, sac, reg \\
\hline & L129 & - & - & $\mathrm{ND}$ & - & NVL & - & - & - & - & NVL & - & - & - & - & NVL & - & - & ND & - & NVL & - & - & ND & - & NVL \\
\hline & L132 & - & - & ND & - & NVL & - & - & ND & - & NVL & - & - & ND & - & NVL & - & - & neuritic PB & - & $\mathrm{nc}$ & - & - & - & - & neuritic dis \\
\hline \multirow{10}{*}{ Group III } & K201 & $2+$ & - & ND & - & NVL & $2+$ & - & $\mathrm{ND}$ & - & prog & - & - & ND & - & static & $4+$ & + & $\mathrm{MB}$ & - & nc, prog, sac & dead & N/A & $\mathrm{ND}$ & dead & dead \\
\hline & K 325 & - & - & $\mathrm{ND}$ & - & NVL & - & - & - & - & NVL & - & - & ND & - & NVL & - & - & ND & - & NVL & - & - & ND & - & NVL \\
\hline & K545 & - & - & ND & - & NVL & - & - & ND & - & NVL & - & - & ND & - & NVL & - & - & ND & - & NVL & - & - & ND & - & NVL \\
\hline & K690 & - & - & ND & - & NVL & - & - & - & - & NVL & - & - & ND & - & NVL & - & - & - & - & NVL & - & - & ND & - & NVL \\
\hline & К 699 & - & - & ND & - & NVL & - & - & - & - & NVL & - & - & ND & - & NVL & - & - & ND & - & NVL & - & - & ND & - & NVL \\
\hline & К 808 & - & - & ND & - & NVL & - & - & ND & - & NVL & - & - & ND & - & NVL & - & - & ND & - & NVL & - & - & ND & - & st \\
\hline & K895 & - & - & $\mathrm{ND}$ & - & NVL & - & - & ND & - & NVL & - & - & ND & - & NVL & - & - & ND & - & st & - & - & ND & - & NVL \\
\hline & K913 & - & - & ND & - & NVL & - & - & ND & - & NVL & - & - & ND & - & NVL & - & - & ND & - & NVL & - & - & & - & NVL \\
\hline & K915 & - & - & ND & - & NVL & - & - & ND & - & NVL & - & - & ND & - & NVL & - & - & ND & - & NVL & - & - & ND & - & NVL \\
\hline & K967 & - & - & ND & - & NVL & - & - & ND & - & NVL & - & - & ND & - & NVL & - & - & ND & - & NVL & - & - & ND & - & NVL \\
\hline \multirow[t]{10}{*}{ Group IV } & J616 & - & - & ND & - & st, NVL & - & - & ND & - & NVL & - & - & ND & - & NVL & - & - & ND & - & NVL & - & - & ND & - & NVL \\
\hline & K 282 & - & - & ND & - & st & - & - & ND & - & NVL & $4+$ & - & ND & - & - & - & - & - & - & st & - & - & ND & - & st \\
\hline & K537 & - & - & ND & - & NVL & - & - & ND & - & NVL & - & - & ND & - & st, NVL & - & - & ND & - & NVL & - & - & ND & + & arthritis \\
\hline & K568 & - & - & ND & - & NVL & - & - & ND & - & NVL & - & - & ND & - & NVL & - & - & ND & - & NVL & - & - & ND & - & NVL \\
\hline & K569 & - & - & ND & - & NVL & - & - & - & - & digit absorb & - & - & ND & - & st & - & - & ND & - & st & - & - & ND & - & NVL \\
\hline & K629 & - & - & ND & - & prog & - & - & $\mathrm{ND}$ & - & prog, st & $4+$ & - & ND & . & - & $4+$ & - & PB & - & prog & $4+$ & + & neuritic MB & + & $\mathrm{nc}$, healing/Rx \\
\hline & K705 & - & - & ND & - & died & - & - & died & $1-$ & died & N/A & N/A & $\mathrm{N} / \mathrm{A}$ & N/A & dead & N/A & $\mathrm{N} / \mathrm{A}$ & N/A & N/A & dead & N/A & N/A & N/A & N/A & dead \\
\hline & K964 & - & - & ND & - & NVL & - & - & $\mathrm{ND}$ & - & NVL & - & - & ND & - & NVL & - & - & ND & - & NVL & - & - & ND & - & NVL \\
\hline & K974 & - & - & ND & - & NVL & - & - & $\mathrm{ND}$ & - & NVL & - & - & ND & - & NVL & - & - & ND & - & NVL & - & - & - & - & NVL \\
\hline & L273 & - & - & ND & - & NVL & - & - & $\mathrm{ND}$ & - & NVL & - & - & ND & - & NVL & - & - & ND & - & NVL & - & - & ND & - & NVL \\
\hline
\end{tabular}

*abbreviations: absorpt, absorption; AFB, acid-fast bacilli; Asp, aspiration; Bx, biopsy; debil, debilitated; Dssm, disseminated; ENL, erythema nodosum leprosum; MB, multibacillary; N/A, not applicable; N-AFB, nasal-acid-fast bacilli; nc, neuritic complications; ND, not done; NVL, no visible leprosy; PB, paucibacillary; prob, probable; prog, progressive disease; reg, regressive disease; resis, leprosy resistant; RM, rhesus monkey; Rx, chemotherapy; sac, sacrificed; st, self-trauma; Suscep, susceptible; wt, weight. 
BCG ONLY GROUP

During the interval of 91-330 days PI, in the group vaccinated and boosted with BCG alone, K948 showed Dssm progressive leprosy at dermal sites; inadvertently, no biopsies were taken, however, and histopathologic documentation of leprosy was not possible in that RM during that period (Table 2). L060 had MB leprosy at inoculation sites. There was no other visible clinical leprosy among the remaining $8 \mathrm{RM}$ in the BCG-only group at this time. Thereafter, over a period of 331-1542 days PI, a total of 3 RM demonstrated histopathologically-documentable leprosy: 1 (L132) with transient neuritic PB during the 851-1197 day PI period, 1 (L060) with initial MB that regressed into neuritic MB and 1 (K948) with transient PB disease during the 851-1197 day interval. A fourth (L123) had histopathology consistent with neuritic leprosy near the TT end of the spectrum, but no AFB could be seen; it was designated as a probable neuritic PB leprosy-positive, but will not be considered as positive histopathologically for calculations. Only one of the 3 (M948) had disease at Dssm dermal (scrotal) sites. L060 and L132 had AFB prominently present within dermal nerves. M948 spontaneously healed, leaving a total of 2 of 10 with persisting histopathologically documentable leprosy and a third RM which failed to show AFB in biopsies in the BCG-only group. Five of the BCG-only-vaccinated group of RM transiently developed AFB-positive nasal secretions over the 331-1197 days PI period, including the 3 dermal biopsy-positive RM. By 1197 days PI, all 10 BCG-only RM were nasal secretion-negative for AFB. There were three self-trauma (st) cases: one of the 3 involved a RM (K477) with no visible signs of leprosy other than a transient nasal AFB-positivity between 561-850 days PI. It was necessary to treat only 2 of the 10 BCG-only-vaccinated RM. There were fewer biopsies taken for study in the vaccinated groups of RM because there were far fewer lesions to biopsy.

These observations show that, as of the present time PI, vaccination/boosting with BCG alone protects RM by $70 \%$ based on AFB-positive histopathology and by at least $50 \%$ based on AFB-positive or -negative histopathology and nasal secretion AFB-positivity. These degrees of protection are significant by the Fisher's exact method ( $p<0.01,70 \%$; and $p<0 \cdot 05,50 \%$ ) compared to 9 of 10 with persisting leprosy in the unvaccinated (positive control) group.

BCG + LD HKML AND BCG + HD HKML GROUPS

Among both of the RM groups vaccinated with BCG + LD and HD HKML, only 3 RM (K201, K282 and K629) became nasal AFB-positive during the 91-850 day period (Table 2). One of the 3 (K282) reverted to nasal-negativity by 851 days PI but continued to selftraumatize. The other 2 AFB-positive RM in these groups developed MB (K201) or neuritic MB (K629) leprosy at dermal inoculation sites. There were no cases of Dssm dermal leprosy among the $20 \mathrm{BCG}+\mathrm{LD}$ and HD HKML vaccine groups of RM. There were $6 \mathrm{RM}$ in these groups with persistent st (Table 2). It was necessary to treat only 2 of the $20 \mathrm{RM}$ in the $\mathrm{BCG}+\mathrm{HKML}$ groups. One additional RM (K201) in these 2 groups was sacrificed due to leprosy complications and another (K705) died of acute gastric dilatation, unrelated to leprosy, to our knowledge.

Altogether, in both groups of BCG + HKML vaccinees, three of $20 \mathrm{RM}$ developed persisting, documentable leprosy. Compared to 9 of 10 unvaccinated RM with persisting leprosy, there was $89 \%$ protection in the BCG + LDHKML group and $78 \%$ protection in the BCG + HDHKML group (statistically significant, $p<0 \cdot 001$, Fisher's exact method). 
Table 3. Clinical results-SMM

\begin{tabular}{|c|c|c|c|c|c|c|c|c|c|c|c|c|c|c|c|c|c|c|c|c|c|}
\hline \multirow[t]{2}{*}{ Days PI } & \multicolumn{6}{|c|}{$210-330$} & \multicolumn{5}{|c|}{$331-510$} & \multicolumn{5}{|c|}{$511-840$} & \multicolumn{5}{|c|}{$841-1170$} \\
\hline & & N-AFB & Dssm & $\mathrm{Bx}$ & $\mathrm{Rx}$ & Notes & $\mathrm{N}$-AFB & Dssm & $\mathrm{Bx}$ & $\mathrm{Rx}$ & Notes & $\mathrm{N}$-AFB & Dssm & $\mathrm{Bx}$ & $\mathrm{Rx}$ & Notes & $\mathrm{N}$-AFB & Dssm & $\mathrm{Bx}$ & $\mathrm{Rx}$ & Notes \\
\hline \multirow[t]{7}{*}{ Group I } & M920 & - & - & MB & - & nc, ENL, reg & - & - & PB & - & nc, reg & - & - & ND & - & spon cure & - & - & ND & - & spon cure \\
\hline & M924 & $2+$ & + & MB & - & nc, prog & - & + & MB & - & reg & $3+$ & + & PB & + & reg & $1+$ & + & neuritic $P B$ & + & $\mathrm{nc}$, healing \\
\hline & M927 & $4+$ & - & $\mathrm{MB}$ & - & nc, digit abs, resis & $4+$ & + & MB & - & $\mathrm{nc}$, prog & $4+$ & + & MB & - & nc, reg & $2+$ & + & ND & + & deform \\
\hline & M930 & $2+$ & + & MB & - & $\mathrm{nc}$, prog & $3+$ & + & $\mathrm{MB}$ & + & nc, prog & - & + & $\mathrm{MB}$ & + & healing on $\mathrm{Rx}$ & - & + & ND & + & healing on $R x$ \\
\hline & M931 & $4+$ & + & MB & - & $\mathrm{nc}$, st, prog & $4+$ & + & MB & + & died on d. 430 & N/A & $\mathrm{N} / \mathrm{A}$ & $\mathrm{N} / \mathrm{A}$ & + & dead & N/A & N/A & N/A & N/A & dead \\
\hline & M942 & - & - & MB & - & st, resis & - & - & ND & - & reg & - & - & PB & - & $\mathrm{nc}$, static & - & - & PB & + & died-neoplasm \\
\hline & M949 & $4+$ & + & MB & - & nc, prog & $4+$ & + & MB & + & neuritic, prog & - & + & MB & + & healing on $\mathrm{Rx}$ & - & + & ND & + & healing on $\mathrm{Rx}$ \\
\hline \multirow[t]{7}{*}{ Group II } & M919 & $4+$ & - & MB & - & reg & $4+$ & - & ND & - & reg & $3+$ & - & MB & - & reg & - & - & ND & + & st, NVL \\
\hline & M928 & $4+$ & + & MB & - & prog, severe & $4+$ & + & MB & + & died on d.518 & N/A & N/A & N/A & + & dead, gastric Asp & N/A & N/A & N/A & + & dead \\
\hline & M929 & - & - & ND & - & NVL & - & - & ND & - & NVL & - & - & $\mathrm{MB}$ & - & prog & $3+$ & + & $\mathrm{MB}$ & + & st, prog \\
\hline & M933 & $4+$ & + & MB & - & $\mathrm{nc}$ & $4+$ & + & MB & + & nc, prog & $4+$ & + & MB & + & healing on $\mathrm{Rx}$ & - & + & ND & + & healing on $\mathrm{Rx}$ \\
\hline & M934 & $2+$ & - & MB & - & prog & - & + & MB & - & reg & - & + & MB & + & nc, static/reg & - & + & ND & + & healing on $R x$ \\
\hline & M935 & - & - & $\mathrm{ND}$ & - & NVL & - & - & ND & - & st, NVL & - & - & ND & - & NVL & - & - & PB & - & st, prog \\
\hline & M936 & - & - & ND & - & uveitis, NVL & - & - & ND & - & NVL & - & - & ND & + & nc, uveitis & - & - & ND & + & sacrificed \\
\hline \multirow[t]{7}{*}{ Group III } & M922 & - & - & PB & - & $\mathrm{nc}$ & - & - & ND & - & $\mathrm{nc}$ & - & - & ND & + & NVL & - & - & ND & - & NVL \\
\hline & M937 & $4+$ & + & MB & - & prog & $4+$ & + & MB & + & prog & $4+$ & + & MB & + & deform. & - & + & ND & + & healing on $\mathrm{Rx}$ \\
\hline & M940 & $4+$ & + & MB & - & nc, prog & $4+$ & + & $\mathrm{MB}$ & + & ENL & - & + & ND & + & healing on $R x$ & - & + & ND & + & healing on $R x$ \\
\hline & M943 & $4+$ & + & MB & - & prog & $4+$ & + & MB & + & ENL & - & + & ND & + & healing on $R x$ & - & + & ND & + & healing on $R x$ \\
\hline & M946 & $4+$ & + & MB & - & prog & $4+$ & + & MB & + & ENL & - & + & ND & + & healing on $R x$ & - & + & ND & + & healing on $\mathrm{Rx}$ \\
\hline & M947 & $2+$ & + & MB & - & prog & $4+$ & + & MB & + & prog & $4+$ & + & MB & + & prog & - & + & ND & + & deform, healing \\
\hline & M951 & $4+$ & + & MB & - & prog & $4+$ & + & MB & - & ENL & $4+$ & + & MB & + & prog & - & + & ND & + & healing on $\mathrm{Rx}$ \\
\hline \multirow[t]{7}{*}{ Group IV } & M923 & $2+$ & + & MB & - & prog & - & + & ND & - & spon.healing & $1+$ & + & $\mathrm{PB}$ & - & neuritic BT-TT & $4+$ & + & ND & + & deform. \\
\hline & M932 & $4+$ & + & MB & - & nc, prog & $4+$ & + & MB & + & ENL, died & N/A & N/A & N/A & N/A & dead & N/A & N/A & N/A & N/A & dead \\
\hline & M938 & $4+$ & + & MB & _ & nc, prog & $4+$ & + & $\mathrm{MB}$ & - & prog & $2+$ & + & MB & + & static/prog & $2+$ & + & ND & + & healing on $\mathrm{Rx}$ \\
\hline & M939 & $3+$ & - & MB & - & nc, prog & $4+$ & - & MB & - & prog & $4+$ & + & $\mathrm{N}$ & + & nc, static/prog & - & + & ND & + & healing on $\mathrm{Rx}$ \\
\hline & M948 & $3+$ & + & MB & - & prog & $4+$ & + & MB & + & ENL & N/A & N/A & N/A & N/A & dead & N/A & N/A & N/A & N/A & dead \\
\hline & M950 & $4+$ & - & MB & - & prog & $4+$ & + & MB & - & prog & $4+$ & + & $\mathrm{MB}$ & + & deform., prog & - & + & ND & + & healing on $\mathrm{Rx}$ \\
\hline & M952 & $3+$ & + & PB & - & prog & $4+$ & + & MB & - & static/prog & $3+$ & + & MB & + & prog & - & + & ND & + & healing on $R x$ \\
\hline
\end{tabular}

abbreviations: absorpt, absorption; AFB acid-fast bacili; Asp, aspiration; Bx, biopsy; debil, debilitated; deform, deformities; Dssm, disseminated; ENL, erythema nodosum leprosum; MB, multibacillary; N/A, not applicable; N-AFB, nasal-acid-fast bacilli; nc, neuritic complications; ND, not done; NVL, no visible leprosy; PB, paucibacillary; prog, progressive disease; reg, regressive disease; resis, leprosy resistant; Rx, chemotherapy; sac, sacrificed; st, self-trauma; spon, spontaneous; Suscep, susceptible; SMM, sooty mangabey monkey; wt, weight. 
CLINICAL OBSERVATIONS IN SMM AFTER CHALLENGE WITH LIVE ML

The clinical results for unvaccinated and the three groups of vaccinated SMM (Table 3, Figure 4) were strikingly different from those observed in RM. At dermal sites of M. leprae challenge, there were indurated lesions two months PI in all 21 unvaccinated (control), BCG alone and BCG + LD HKML groups of SMM; six of the seven BCG + HD HKML group had similar lesions at 2 months. Based on prior studies of experimental leprosy in SMM, it was not surprising that essentially all dermal lesions had BL-LL (MB) histopathology at this time. $^{13-15}$ These lesions are not considered to be entirely indicative of the clinical susceptibility status of the animals, but rather, due in part to the dermal inoculation of relatively high numbers of $M$. leprae locally into a species that is inherently more susceptible to MB forms of leprosy than RM. ${ }^{14,15}$ Beginning at 4 months PI, some of the lesions began to spontaneously disappear or diminish in some animals, progress in others and disseminate to uninoculated sites (including the nasal mucosa) in many SMM (Table 3).

By 7 months (210 days) PI, lesions had cleared in 3 of the 7 BCG animals (M929, M935 and M936). Thus, we report in detail data beginning at 210 days PI, a point at which resistant SMM had begun to distinguish themselves clinically from susceptible animals. We consider the relative status of groups 210 days PI and thereafter as being relevant to and reflective of possible anti- $M$. leprae immunization/vaccination. By 210 days PI, all SMM in the unvaccinated and the BCG + HKML groups were histopathologically leprosy-positive, compared to 4 in the BCG-only vaccinated group (Figure 4). By 800 days PI, 2 additional BCG-only SMM became leprosy-positive (Figure 4), for a total of 6 leprosy-positive SMM in this group over the course of study. The details of disease progression in the SMM groups beginning at 210 days PI are as follows (Table 3).

UNVACCINATED GROUP

During the 210-330 days PI period, all 7 unvaccinated SMM had MB lesions at sites of

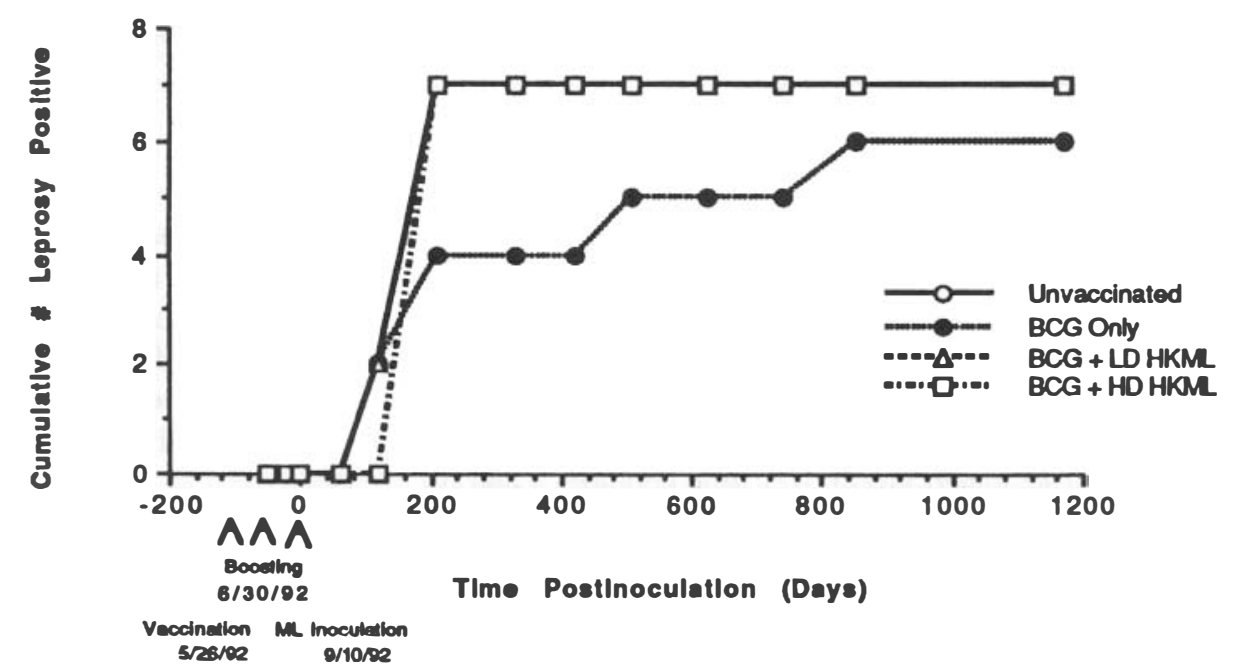

Figure 4. Cumulative number of leprosy-positive SMM, based on biopsy results using Ridley-Jopling classifications of infiltrations of AFB-positive dermal lesions, in the unvaccinated or vaccinated groups over the course of observation after live $M$. leprae challenge. 
dermal inoculation, 4 had lesions at Dssm dermal sites and 5 had AFB-positive nasal secretions. Between 420 and 510 days PI, 3 of the 7 SMM in this group were placed on multidrug therapy (MDT, rifampicin, clofazimine, dapsone) due to the progression of clinical disease (Table 3; Figure 4). Eventually, over the course of observation, spanning 1170 days, 6 of the unvaccinated group were treated due to Dssm or localized (M942) leprosy and one spontaneously cured (M920). M931 died on day 430 PI, one day after MDT initiation, presumably due to the severity of the disease. M942 developed a liver neoplasm at near 1170 days PI and was humanely sacrificed.

Altogether, six unvaccinated SMM developed neuritic complications (nc) such as nerve edema or physical deformities, along with other persisting symptoms of leprosy; 5 developed M. leprae disease and one developed PB leprosy (M942) (the 7th SMM, M920, developed the only case of ENL in this group, during the 210-330 days PI period and subsequently spontaneously healed of all leprosy signs).

\section{BCG ONLY GROUP}

The disease progression in the BCG group was slower in that, during the 210-330 days PI period, lesions were smaller, less numerous and 4 BCG-vaccinated SMM had MB leprosy (2 with Dssm dermal leprosy together with AFB-positive nasal smears) (Table 3; Figure 4). This approached significance (Fisher's exact method, $p=0.09$ ) compared to 7 cases of M. leprae leprosy (4 Dssm and AFB-positive nasal secretions) in the unvaccinated group during the 210-330 days PI period (Table 3). During the 331-510 days PI period, 2 BCG-only vaccinated SMM (M928 \& M933) were placed on chemotherapy (Table 3). M928 died soon thereafter due to leprosy. During the 841-1170 days PI period, M935 newly-developed PB leprosy at sites of ML inoculation resulting in the loss of statistical significance relative to the control group. By 1170 days PI, there were $5 \mathrm{MB}$ cases, one PB case and one SMM (M936) with little or no visible leprosy (NVL) except uveitis, requiring chemotherapy. M928 died of causes unrelated to leprosy approximately six months after the initiation of treatment.

There were 3 SMM with nc and no SMM with ENL in the BCG-only vaccinated group of SMM (M933, M934 \& M936) (Table 3).

BCG + LD HKML GROUP

During the period of 210-330 days PI, 6 SMM in this group were positive for Dssm MB leprosy and one for PB leprosy at dermal inoculation sites (M922). All but the PB case were nasal secretion-positive for AFB (Table 3). The PB case went on to spontaneously heal. The other 6 were successfully placed on chemotherapy by 840 days due to the rapid progression, continued Dssm and exacerbation of the clinical symptoms. The number and severity of the lesions became significantly greater ( $p<0 \cdot 05$, Mancova) in the BCG + LD HKML group than in the unvaccinated group over time (Figures 5 and 6).

Two cases of nc were observed in this group of SMM over the course of this study; four cases of ENL (M940, M943, M946 and M951) were observed by 510 days PI.

BCG + HD HKML GROUP

All seven SMM in the BCG + HD HKML group were nasal-positive for AFB between 210 and 330 days PI and all were histopathologically positive for dermal leprosy (6 MB and 1 
PB). All but one SMM (M939) showed Dssm by 510 days PI. By the 841-1170 days PI interval, all 7 SMM in the BCG + HD HKLM group had developed Dssm lesions; 4 of the 7 HD HKML group had developed physical signs of nc. Before 840 days PI, all 7 BCG + HD HKML SMM were either on chemotherapy or (two) had died secondary to ENL episodes (Table 3).

Similar to the BCG + LD HKML group, leprosy progressed much more rapidly and, with time, became visibly more severe in the BCG + HD HKML group than in the unvaccinated group (Figures 5 and 6). The average number of lesions per monkey was greatest in the unvaccinated, control SMM group over a period of 100-450 days PI (Figure 5). Thereafter, the average number of lesions spontaneously decreased in the unvaccinated group while the number of lesions continued to increase significantly in the BCG + HKML groups of SMM over the ensuing 300 days of observation (Figure 5). Over the first 200 days PI, the average lesion size was similar in all 4 groups of SMM; by 310 days PI, the average lesion size was greatest in the control group, but continued to increase in the BCG + HKML groups; and by 720 days PI, the average lesion size was significantly greatest $(p<0 \cdot 05$, Mancova) in the $\mathrm{BCG}+\mathrm{HKML}$ groups and had diminished in the unvaccinated and the BCG only groups (Figure 6). The BCG-only group maintained essentially the lowest average number of lesions and the lowest average lesion size over the course of our observations, but there was no statistical significance (Figures 5 and 6).

PHENOLIC GLYCOLIPID-I ANTIGEN LEVELS IN SERA OF VACCINATED AND UNVACCINATED RM AND SMM

There was no detectable PGL-I antigen found in sera from any of the RM in this study (data not shown). Readily detectable levels of PGL-I antigen were found in sera of the four groups of SMM (Figure 7). Compared to the unvaccinated group, PGL-I antigen levels reached

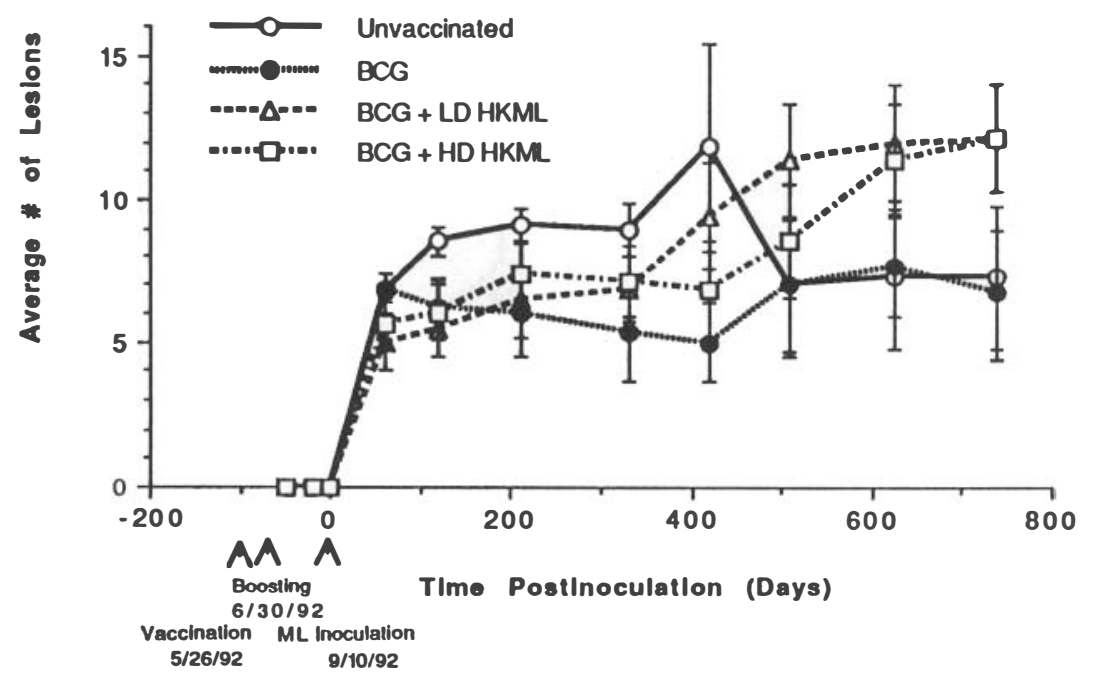

Figure 5. Average number of lesions (+/-1 SEM) observed longitudinally after challenge of SMM groups with live M. leprae. 


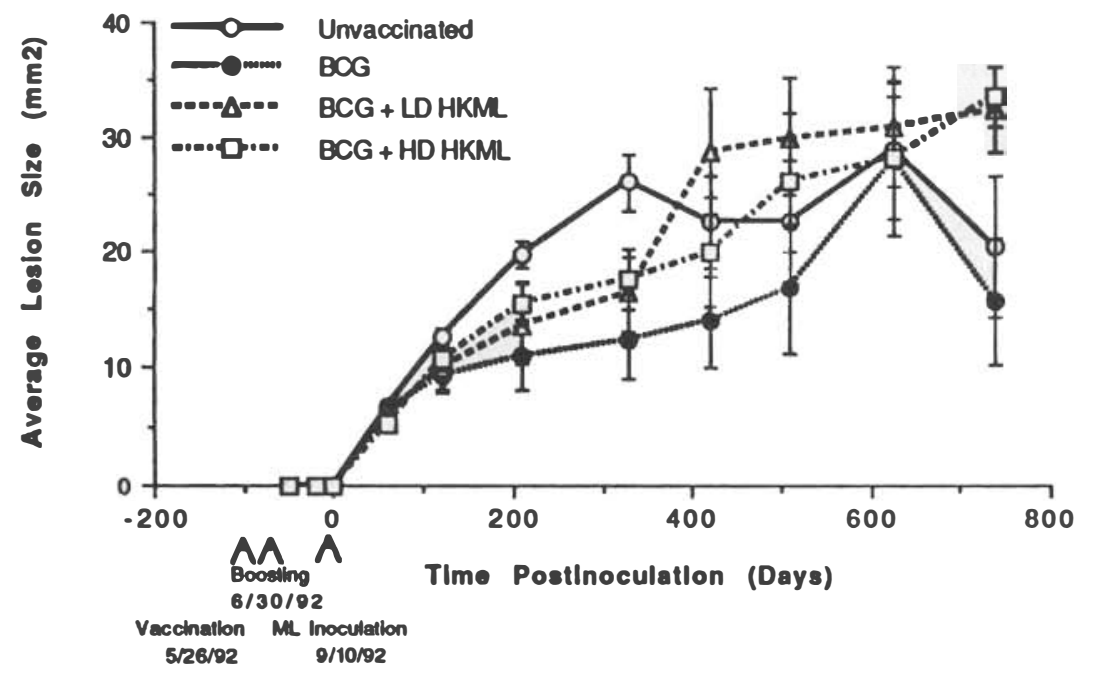

Figure 6. Average lesion size (+/- 1 SEM) observed longitudinally after challenge of SMM groups with live M. leprae.

statistically significant higher maximal levels $(p<0 \cdot 0025$, Mancova $)$ in the SMM BCG + LD HKML group, but not in the BCG + HD HKML group; the PGL-I levels rose more slowly and attained lower maximal levels in the BCG only group, but was not significantly different from controls.

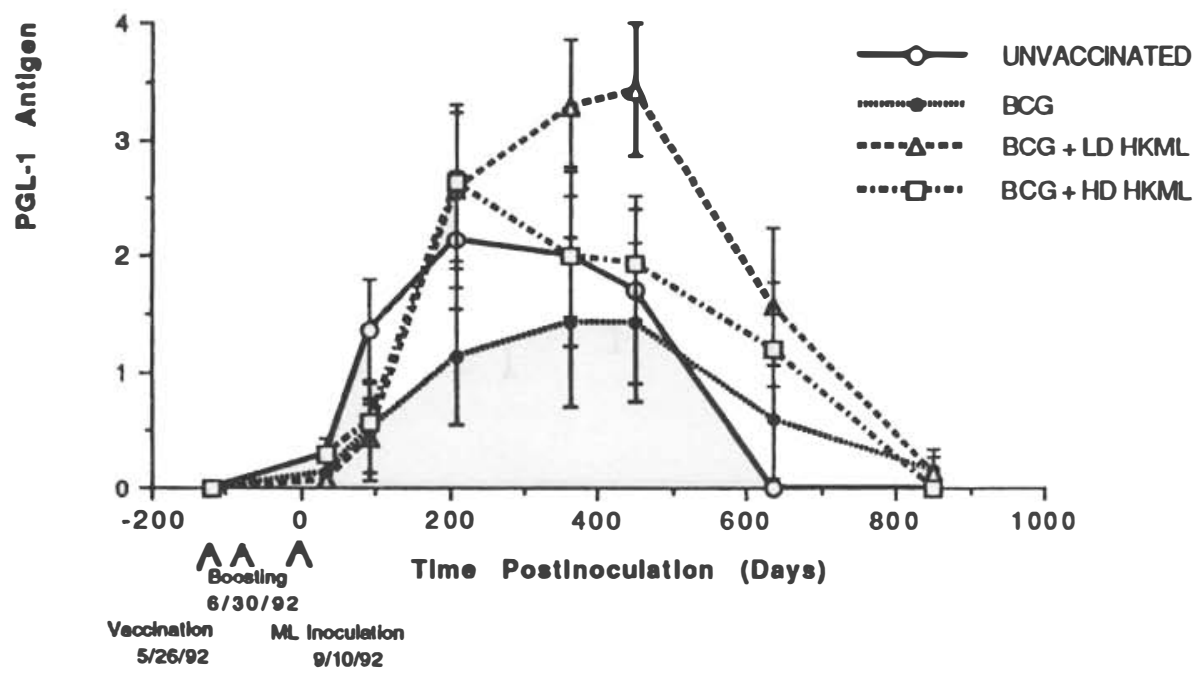

Figure 7. Average PGL-I antigen levels (+/- 1 SEM) in serum samples obtained longitudinally prior to vaccination, after vaccination, after boosting and at intervals after challenge with live M.leprae in SMM groups. 


\section{Discussion}

The vaccination results in RM, which, as a species, generally develop PB forms of leprosy in approximately $80 \%$ of cases, show that BCG alone offers $70 \%$ protection of RM against clinical leprosy, based on lesion bioposy histopathologic results spanning 3.5 years after challenge with live M. leprae. A total of 3 of the 10 BCG-only-vaccinated RM had biopsydocumentable leprosy and another had mononuclear cell infiltration, but no AFB were present. Five of the 10 BCG-only-vaccinated RM, including the 3 biopsy-positives, had transient episodes of AFB-positivity in nasal secretions. In the SMM species, which generally develop MB forms of leprosy in over $80 \%$ of cases, ${ }^{12-15,18,22,24}$ however, the results show that there is no statistically significant decrease in the total numbers of SMM with sustained histopathologically-documentable leprosy in the long term (approximately 3.5 years) with BCG alone. SMM vaccinated with BCG alone developed disease more slowly with fewer and smaller lesions. There was a lowered PGL-I antigen level in the serum of SMM in the BCGvaccinated group over most of the time of observation, consistent with a presumably lowered bacterial burden, but this was not statistically significant compared to unvaccinated controls.

We used the same mixtures of batches of BCG, all of the Glaxo strain, to vaccinate both the RM and SMM; similar numbers of live ML with similar MI's were used to challenge both the RM and SMM; the inocula were from the same original source and each ML batch had been subpassaged from the same SMM through an armadillo for monkey inoculations. The major obvious difference between the RM and SMM study was the monkey species. In view of the close phylogenetic and immunologic similarities between RM, SMM and humans, the results strongly suggest that the ability of BCG to protect populations against clinical leprosy depends on the susceptibility of a given population to MB vs PB leprosy, which is known to vary among humans geographically. Thus, the data offer at least one explanation for variations in previous studies of antileprosy protective effects of BCG vaccination. ${ }^{1,3-5,25}$ The prior studies in Uganda, Burma, New Guinea, south India and Malawi showed a variation from 20 to $80 \%$ protection by BCG alone. The majority of leprosy cases in those regions are non-lepromatous forms, but the exact make-up of clinical populations and the natural histories of disease and ratios of types of disease vary from one region to the other. ${ }^{6} \mathrm{~A}$ human study in southern Viet Nam, where there is a variable range of 30-70\% lepromatous cases, depending on the region, concluded that BCG offered protection against nonlepromatous leprosy but had no protective effect against lepromatous leprosy. ${ }^{7}$ Our results are consistent with those conclusions, but suggest that the speed of progression of symptoms and the severity or degree of disease symptoms may be lessened in MB-prone patients by vaccination with BCG alone. At any rate, the data herein suggest that BCG alone is statistically effective in protection of many individuals from leprosy and is safe to use as a vaccine; at worst BCG may provide little or no protection against leprosy in some individual recipients. $B C G$ has been convincingly shown to protect human populations in Malawi $i^{6,25}$ and elsewhere. ${ }^{1-5,7}$

The protective efficacy of BCG alone as a vaccine was enhanced in RM by the addition of HKML together with BCG. A recent follow-up report from Malawi showed a reduced risk of leprosy in humans under the age of 15 vaccinated with $\mathrm{BCG}+$ killed $M$. leprae compared to BCG alone, but overall, in all age groups, there was no improved benefit by the addition of killed $M$. leprae to the effective $\mathrm{BCG}$ vaccine.

Contrary to the RM results, vaccination of MB leprosy-prone SMM with BCG + HKML caused the disease to become more severe clinically and increased the rate and degree of 
progress to the point of threatening the lives of the animals if not quickly placed on chemotherapy at the outset of observed dissemination and progressive clinical symptoms. Serum PGL-I levels are presumably proportional to the bacterial load; ${ }^{12,23}$ significantly higher PGL-I levels in the SMM BCG + LD HKML group and lower levels (not statistically significant) in the BCG only group compared to controls are, therefore, consistent with the clinical observations indicating that BCG + LD HKML renders SMM more susceptible to the progression of the disease and BCG alone may at least slow the progression and/or degree of MB disease in SMM. Compared to the unvaccinated group, PGL-I levels in the BCG + HD HKML-vaccinated SMM failed to significantly correlate with the clinically observed increased susceptibility to LL leprosy in that group, contrary to expectations. These observations suggest that the immune mechanisms of leprosy susceptibility are complex, that immune responses to vaccination are dose-dependent and do not necessarily directly correlate proportionately with effects on clinical leprosy susceptibility. The absence of detectable levels of PGL-I antigen in the sera of RM from unvaccinated or vaccinated groups is consistent with our clinical observations that RM, as a species, are more resistant than SMM to MB forms of leprosy.

The results suggest that caution should be exercised in the use of HKML in BCG vaccine preparations for use in human populations since it is not possible to determine a priori which humans will be susceptible to $\mathrm{MB}$ vs $\mathrm{PB}$ forms of leprosy. In consideration of our observations and of the demonstrated failure of killed $M$. leprae to add significantly to BCG's protective efficacy in humans, ${ }^{2}$ it appears prudent to avoid the use of killed M. leprae altogether in BCG vaccines.

We do not yet know an exact explanation for the opposing results in RM vs SMM in protective ability of HKML together with BCG other than the species difference in susceptibility towards PB vs MB forms of leprosy. Comparative longitudinal immunologic studies on these RM and SMM have provided some clues, however, as to some aspects of the immune mechanisms involved in the differences we have observed and will be reported elsewhere (Gormus, BJ et al, p. 24).

\section{Acknowledgments}

We acknowledge the expert technical assistance of the following persons: Ms's Cynthia Trygg, Carolyn Coyne, Doris O’Leary, Renee Grow, Eva Pecunia and Mr Calvin Lanclos. We thank Ms Ann Bennett for her secretarial contributions. Presented at the 95th annual meeting of the American Society for Microbiology, Washington, DC 21-25 May, 1995 (abstract \# U114). Supported by grants \#AI-19301 from the National Institutes of Allergy and Infectious Diseases (NIAID) and \#RR-00164 from the National Center for Research Resources (NCRR).

\section{References}

${ }^{1}$ Bechelli LM, Lwin K, Gallego-Garbajosa PG, et al. J. BCG Vaccination of children against leprosy: nine-year findings of the controlled WHO Trial in Burma. Bull WHO 1974; 51: 93-99.

2 Convit J, Sampson C, Zuniga M, et al. Immunoprophylactic Trial with Combined Mycobacterium leprae/BCG Vaccine Against Leprosy: Preliminary Results. The Lancet, 1992; 339: 446-450.

${ }^{3}$ Scott GC, Russell DA, Boughton CR, Vincin DR. Untreated leprosy: probability of shifts in Ridley-Jopling 
classification. Development of 'flares' or Disappearance of Clinically Apparent Disease. Int J Lepr, 1978; 44: $110-122$.

4 Stanley SJ, Howard C, Stone MM, Sutherland I. BCG Vaccination of Children Against Leprosy in Uganda: Final Results. J Hygiene (Cambridge) 1981; 87: 233-248.

5 Tripathy SP. The Case for BCG. Ann Nat Acad Med Sci (India), 1983; 19: 12-21.

${ }^{6}$ Fine PEM. BCG Vaccination Against Tuberculosis and Leprosy. Brit Med Bull, 1988; 44: 691-703.

7 Abel L, Cua VV, Oberti J, Lap VD, Due LK, Grosset J, Lagrange PH. Leprosy and BCG in Southern Viet Nam. The Lancet, 1990; 335: 1536.

8 Palmer CE, Long MW. Effects of Infection with Atypical Mycobacteria on BCG Vaccination and Tuberculosis. Amer Rev Resp Dis, 1966; 94: 553-568.

9 Palmer CE, Edwards LB. Identifying the Tuberculous Infected. J Amer Med Assn, 1968; 205: 167-169.

10 Guld J. BCG as an Immunizing Agent. Vol. 14 Washington, DC: DHEW, 1971 (Chamberlayne EC, ed. Status of Immunization in Tuberculosis in 1971. Fogerty International Centennial Proceedings).

11 Willis S, Vandiviere M. The Heterogeneity of BCG. Amer Rev Resp Dis, 1961; 84: 288-290.

12 Gormus BJ, Ohashi DK, Ohkawa S, Walsh G, Meyers WM, Brennan PJ, Trygg C. Serologic Responses to Mycobacterium leprae-Specific Phenolic Glycolipid I Antigen in Sooty Mangabey Monkeys with Experimental Leprosy. Int J Lepr, 1988; 56: 537-545.

13 Gormus BJ, Xu K, Meyers WM, Walsh GP, Levis WR, Meeker HC. Antibodies to Lipoarabinomannan Antigen in Sooty Mangabey Monkeys Experimentally Inoculated with Mycobacterium leprae. Int J Lepr, 1990; 58: 65-72.

14 Gormus BJ, Xu K, Baskin GB, et al. Experimental Leprosy in Monkeys. I. Sooty Mangabey Monkeys: Transmission, Susceptibility, Clinical and Pathological Findings. Lepr Rev, 1995; 66: 96-104.

15 Gormus BJ, Xu K, Baskin GB, et al. Experimental Leprosy in Monkeys. II. Longitudinal Serological Observations in Sooty Mangabey Monkeys. Le pr Rev, 1995; 66: 105-125.

16 Meyers WM, Walsh GP, Brown HL, et al. Leprosy in a Mangabey Monkey: Naturally-acquired Infection. Int $J$ Lepr, 1985; 53: 1-14.

17 Wolf RH, Gormus BJ, Martin LN, Walsh GP, Meyers WM, Binford CH. Experimental Leprosy in Three Species of Monkeys. Science, 1985; 227: 529-531.

18 Draper P. Purification of M. leprae. Protocol 1/79. Geneva: IMMLEP Steering Committee, WHO, 1979.

19 Shepard CC, McRae DH. A Method for Counting Acid Fast Bacteria. Int J Lepr, 1968; 36: 78-82.

20 Ridley DS, Jopling WH. Classification of Leprosy According to Immunity: A Five Group System. Int J Lepr, 1966; 34: 255-273.

${ }^{21}$ Baskin GB, Wolf RH, Gormus BJ, et al. Experimental Leprosy in the Mangabey Monkey (Cercocebus atys): Necropsy Findings. Int J Lepr, 1985; 53: 269-277.

22 Baskin GB, Gormus BJ, Martin LN, et al. Experimental Leprosy in a Rhesus Monkey: Necropsy Findings. Int $J$ Lepr, 1987; 55: 109-115.

${ }^{23}$ Cho S-N, Hunter SW, Gelber RH, Rea TH, Brennan PJ. Quantification of the Phenolic Glycolipid of Mycobacterium leprae and Relevance to Glycolipid Antigenemia in Leprosy. J Infect Dis, 1986; 153: 560-569.

${ }^{24}$ Fine PEM, Ponnighaus JM, Maine N, Clarkson JA, Bliss L. Protective Efficacy of BCG Against Leprosy in Northern Malawi. The Lancet 1986; II: 449-502.

25 Fine PEM, Clayton D, Ponnighaus JM, Warndorf DK. Randomized Controlled Trial of Single BCG, Repeated BCG, or Combined BCG and Killed Mycobacterium leprae Vaccine for the Prevention of Leprosy and Tuberculosis in Malawi. The Lancet 1996; 348: 17-24. 